\title{
Costs of Acute Headache Medication Use and Productivity Losses Among Patients with Migraine: Insights from Three Randomized Controlled Trials
}

\author{
Joshua K. Porter ${ }^{1}\left[\right.$ [ $\cdot$ Gian Luca Di Tanna ${ }^{1} \cdot$ Richard B. Lipton ${ }^{2} \cdot$ Sandhya Sapra $^{3} \cdot$ Guillermo Villa $^{1}$
}

Published online: 30 October 2018

(c) The Author(s) 2018

\begin{abstract}
Background Migraine is associated with a substantial physical and emotional burden for patients. There is also a large economic burden associated with migraine, in terms of lost productivity and healthcare resource use. By reducing the number of monthly migraine days (MMD) experienced by patients, effective preventive treatments can reduce acute medication use and costs of lost productivity.

Methods Patient level data from three erenumab clinical trials (NCT02456740, NCT02483585 and NCT02066415) were combined and migraine day frequencies were examined. The number of days per month on which patients used acute medication was estimated as a function of MMD. Productivity losses were estimated based on patient responses to the Migraine Disability Assessment questionnaire. Zero-inflated Poisson regression models were used to predict acute medication use and productivity losses per MMD.

Results The results demonstrated that as MMD increased, use of acute medication also increased. Similarly, as MMD increased, loss of productivity (due to absenteeism and presenteeism) also increased. The relationship of MMD to both acute headache medication use and lost productivity was non-linear, with marginal outcomes increasing with frequency. Conclusions As MMD increased, acute medication use and productivity loss also increased, but the relationship was nonlinear. Therefore, it is important that the distribution of MMD patients is accounted for when estimating the outcomes of migraine patients. By reducing the MMD experienced by patients, effective preventive agents may reduce the requirement for acute medication and also reduce productivity loss, which may translate into potential economic savings.
\end{abstract}

Electronic supplementary material The online version of this article (https://doi.org/10.1007/s41669-018-0105-0) contains supplementary material, which is available to authorized users.

Joshua K. Porter

joshua.porter@amgen.com

1 Economic Modeling Center of Excellence, Amgen (Europe) GmbH, Suurstoffi 22, Rotkreuz, Zug 6343, Switzerland

2 Albert Einstein College of Medicine, New York, NY 10461, USA

3 Amgen Inc, Thousand Oaks, CA 91320, USA

\section{Key Points for Decision Makers}

An increase in migraine day frequency was associated with an increase in acute medication use and productivity loss, but this relationship was not linear.

The accurate estimation of acute medication costs and productivity losses is important to assess economic benefits that might be achieved with successful migraine preventive therapies.

\section{Introduction}

Migraine is a neurological disorder that causes persistent and debilitating headaches, often accompanied by additional neurological symptoms [1]. Migraine without aura is defined as the occurrence of at least five attacks lasting 
4-72 h (untreated or unsuccessfully treated) with at least two of four pain features (unilateral, throbbing, moderate to severe pain intensity, exacerbation with physical activity) and one of two associated symptom profiles (nausea or vomiting or photophobia and phonophobia) [2].

As migraine is a long-term disorder with episodic attacks [3], people with the condition experience days with and without headaches. The condition is divided into episodic migraine (EM; 0-14 headache days per month [defined as 28 days]) and chronic migraine (CM; $\geq 15$ headache days per month for at least 3 months, $\geq 8$ of which meet the criteria for migraine and/or respond to migraine-specific treatments), and migraine classification may guide the treatment choices available to patients $[2,4,5]$.

Migraine is associated with both high direct healthcare costs and indirect costs due to lost workplace productivity [6-8]. Indirect costs arise from full days of productive work lost (absenteeism) and days worked with diminished productivity (presenteeism). These indirect costs are estimated to account for $50-70 \%$ of the total costs of migraine [9]. Migraine prevention aims to reduce the frequency, severity, and duration of attacks, which are associated with high costs to patients, employers, and healthcare systems $[10,11]$. Effective preventive agents reduce the number of monthly migraine days (MMD) and, consequently, reduce acute treatment costs and patient disability [12].

Erenumab is a fully human monoclonal antibody that specifically blocks the calcitonin gene-related peptide receptor complex $[13,14]$. Erenumab is approved by the Food and Drug Administration (FDA) for the prevention of migraine in adults [15], and has been shown to significantly reduce the number of MMD in patients with EM and CM compared with placebo $[14,16,17]$. The erenumab clinical studies included endpoints which recorded the use of acute medication (AM) and the impact of migraine on patients' productivity. Here, we assess how the level of AM use and workplace productivity losses are affected by differences in MMD during the double-blind period in clinical studies.

\section{Methods}

\subsection{Population}

The study involves the patient populations of three pivotal clinical studies of erenumab: the phase 3 NCT02456740 (STRIVE) study (EM; $n=955$ ), the phase 3 NCT02483585 (ARISE) study (EM; $n=577)$, and the phase 2 NCT02066415 study (CM; $n=667)$ [14, 16, 17]. The EM studies recruited patients with 4-14 MMD and headache days, and the CM study recruited patients with $\geq 15$ headache days, of which $\geq 8$ were migraine days. The STRIVE, ARISE and CM studies recruited in 13, eight, and ten countries across North America and Europe, respectively, and all were conducted between 2014 and 2016.

Patient-level data from the placebo and erenumab (70 $\mathrm{mg}$ and $140 \mathrm{mg}$ ) arms of the clinical studies were pooled to generate one complete migraine data set containing the full range of MMD. Besides the MMD eligibility criteria, patient characteristics in the studies were comparable. Due to differences in study durations, data were available for up to 24 weeks for patients with EM and 12 weeks for those with CM.

\subsection{Acute Headache Medication Use}

The clinical studies included endpoints reporting the number of days on which migraine-specific AM (triptans and ergotamine derivatives) was used by participants in each 28-day visit period. The number of days on which patients used any headache AM was also recorded. These data were used to derive the number of days on which patients used non-migraine-specific AM (all headache AM minus migraine-specific $A M)$, such that two mutually exclusive AM types (migraine-specific and non-migraine-specific) could be modelled.

To understand how MMD is associated with the use of AM across the cohort and duration of the studies, the reported number of days of AM use was regressed against MMD, employing a set of covariates, including age, sex, race, treatment group, prior failed preventive migraine medication status, and MMD at study baseline. Covariates were selected based on known associations and clinical advice $[6,18]$.

As the response variables are count data, and given the considerable proportion of patients who reported no AM use, zero-inflated Poisson regression models were determined as the most appropriate to assess two AM use outcomes independently: days on which patients reported using migraine-specific AM and days on which patients reported using non-migraine-specific AM. To account for the repeated measurements and to minimize the risk of type-1 error, robust standard errors at the patient level were estimated. Predicted mean AM use by MMD was estimated using the Delta method [19].

Costs associated with the two types of AM use were estimated using 2018 Wholesale Acquisition Costs from AnalySource and published data on the types and share of different medications used for EM and CM (see Online Resource 1 in the electronic supplementary material). The calculation assumed average costs per day of migraine-specific AM of US\$7.52 and US\$8.55 for EM and $\mathrm{CM}$, respectively, and costs per day of non-migrainespecific AM of US\$1.58 and US\$2.44 for EM and CM, respectively. 


\subsection{Productivity Loss}

The clinical studies also assessed patients' productivity losses, using the Migraine Disability Assessment (MIDAS) questionnaire, a migraine-specific instrument used to record the impacts of migraine on patients' productive time and social functioning $[20,21]$. Two outcomes are typically considered when investigating the economic impact of lower productivity among patients with migraine: absenteeism and presenteeism. Absenteeism is defined as the number of days on which a patient misses work or study altogether; presenteeism is the number of days on which productivity is reduced by at least $50 \%$ (but not qualifying as absenteeism days). In the MIDAS, these outcomes are measured using responses to two questions: (1) On how many days in the last month did you miss work or school because of your headaches? (absenteeism); and (2) On how many days in the last month was your productivity at work or school reduced by half or more because of your headaches? (presenteeism).

In the CM study, the original MIDAS was administered with a 3-month recall period. In the two EM studies, a modified monthly MIDAS, including a 4-weekly recall period (i.e. responses related to the previous 28 days, as per the questions above), was used. Therefore, to combine the study data sets, 3-monthly observations from the CM study were divided into monthly observations. To do this, it was assumed that the distribution of absenteeism and presenteeism days across the 3 months was the same as the distribution of migraine days (for which monthly observations were collected).

Zero-inflated Poisson regression models were fitted with the number of absenteeism and presenteeism days as response variables and regressed against MMD using the covariates set described previously. As for the analysis of AM use, the zero-inflated models were selected due to the high proportion of patients who reported no days of lost productivity. As before, robust standard errors were generated and predicted values of absenteeism and presenteeism days for each MMD level were estimated using the Delta method [19].

Lost productivity costs were calculated from this output, assuming 8 working hours per day and a median hourly wage of US\$26.00 (valued using the 2018 US dollar rate) [22]. The total cost of lost productivity for an absenteeism day was US\$208.00 and, assuming a minimum 50\% productivity loss on presenteeism days, the total cost of lost productivity for a presenteeism day was US\$104.00.

\section{Results}

\subsection{Patient Characteristics}

Patient characteristics are based on those of the three erenumab clinical studies (Table 1). In all three studies, the majority of patients were female and white. With regard to clinical characteristics, the trial populations are

Table 1 Patient characteristics at baseline

\begin{tabular}{|c|c|c|c|c|c|c|c|c|}
\hline \multirow[t]{2}{*}{ Characteristic } & \multicolumn{3}{|c|}{$\begin{array}{l}\text { Episodic migraine (NCT02456740) } \\
\text { STRIVE [14] }\end{array}$} & \multicolumn{2}{|c|}{$\begin{array}{l}\text { Episodic migraine } \\
\text { (NCT02483585) } \\
\text { ARISE [16] }\end{array}$} & \multicolumn{3}{|c|}{$\begin{array}{l}\text { Chronic migraine (NCT02066415) } \\
\text { [17] }\end{array}$} \\
\hline & Placebo & $\begin{array}{l}\text { Erenumab } \\
70 \mathrm{mg}\end{array}$ & $\begin{array}{l}\text { Erenumab } \\
140 \mathrm{mg}\end{array}$ & Placebo & $\begin{array}{l}\text { Erenumab } \\
70 \mathrm{mg}\end{array}$ & Placebo & $\begin{array}{l}\text { Erenumab } \\
70 \mathrm{mg}\end{array}$ & $\begin{array}{l}\text { Erenumab } \\
140 \mathrm{mg}\end{array}$ \\
\hline Number of patients & 319 & 317 & 319 & 291 & 286 & 286 & 191 & 190 \\
\hline Mean age (SD), years & $41.3(11.2)$ & $41.1(11.3)$ & $40.4(11.1)$ & $42.2(11.5)$ & $42.3(11.4)$ & $42.1(11.3)$ & $41.4(11.3)$ & $42.9(11.1)$ \\
\hline \multicolumn{9}{|l|}{ Sex, $n(\%)$} \\
\hline Male & $45(14.1)$ & $49(15.5)$ & $47(14.7)$ & $44(15.1)$ & $41(14.3)$ & $60(21.0)$ & $25(13.1)$ & $30(15.8)$ \\
\hline Female & $274(85.9)$ & $268(84.5)$ & $272(85.3)$ & $247(84.9)$ & $245(85.7)$ & $226(79.0)$ & $166(86.9)$ & $160(84.2)$ \\
\hline \multicolumn{9}{|l|}{ Race, $n(\%)$} \\
\hline White & $277(86.8)$ & $281(88.6)$ & $293(91.8)$ & $259(89.0)$ & $259(90.6)$ & $268(93.7)$ & $176(92.1)$ & $184(96.8)$ \\
\hline Black & $24(7.5)$ & $24(7.6)$ & $18(5.6)$ & $27(9.3)$ & $24(8.4)$ & $11(3.8)$ & $10(5.2)$ & $6(3.2)$ \\
\hline Other & $18(5.6)$ & $12(3.8)$ & $8(2.5)$ & $5(1.7)$ & $3(1.0)$ & $7(2.4)$ & $5(2.6)$ & $0(0.0)$ \\
\hline Mean MMD (SD) & $8.2(2.5)$ & $8.3(2.5)$ & $8.3(2.5)$ & $8.4(2.6)$ & $8.1(2.7)$ & $18.2(4.7)$ & $17.9(4.4)$ & $17.8(4.7)$ \\
\hline Mean MHD (SD) & $9.3(2.6)$ & $9.1(2.6)$ & $9.3(2.5)$ & $9.3(1.7)$ & $9.1(2.7)$ & $21.1(3.9)$ & $20.5(3.8)$ & 20.7 (3.8) \\
\hline $\begin{array}{l}\text { Patients with } \geq 1 \text { prior } \\
\text { failed preventive, } n(\%)\end{array}$ & $127(39.8)$ & $127(40.1)$ & $116(36.4)$ & $114(39.2)$ & $115(40.2)$ & $200(69.9)$ & $127(66.5)$ & $126(66.3)$ \\
\hline
\end{tabular}

ARISE A phase 3, RandomIsed, double-blind, placebo-controlled Study to Evaluate the efficacy and safety of AMG 334 in migraine prevention, $N C T$ national clinical trial, $M H D$ monthly headache days, $M M D$ monthly migraine days, $S D$ standard deviation, STRIVE Study to evaluate the efficacy and safety of erenumab in migraine prevention 
generalizable to patients for whom prevention is considered appropriate ( $\geq 4 \mathrm{MMD})$.

\subsection{Acute Medications}

Predicted AM use by MMD is presented in Fig. 1a and Online Resource 2. Details of the regression model are presented in Online Resource 4. A patient experiencing 8 MMD is predicted to use migraine-specific AM on 2.82 (95\% confidence interval [CI] 2.80-2.85) days and nonmigraine-specific AM on 2.75 (95\% CI 2.74-2.77) days per month. This medication use is expected to cost a mean of
US\$21.22 (range US\$21.06-US\$21.39) and US\$4.34 (range US\$4.32-US\$4.37) per month, respectively. A patient with CM experiencing $18 \mathrm{MMD}$ is predicted to use migrainespecific AM on 7.06 (95\% CI 7.01-7.12) days and nonmigraine-specific AM on 4.81 (95\% CI 4.79-4.84) days per month. This medication use is expected to cost a mean of US\$60.40 (range US\$59.92-US\$60.87) and US\$11.75 (range US\$11.68-US\$11.81) per month, respectively. The analysis shows that the number of both migraine-specific and non-migraine-specific AM use days increases progressively for each incremental migraine day. The results indicate a
Fig. 1 a Acute medication use by monthly migraine days. $\mathbf{b}$ Productivity losses by monthly migraine days
(A)

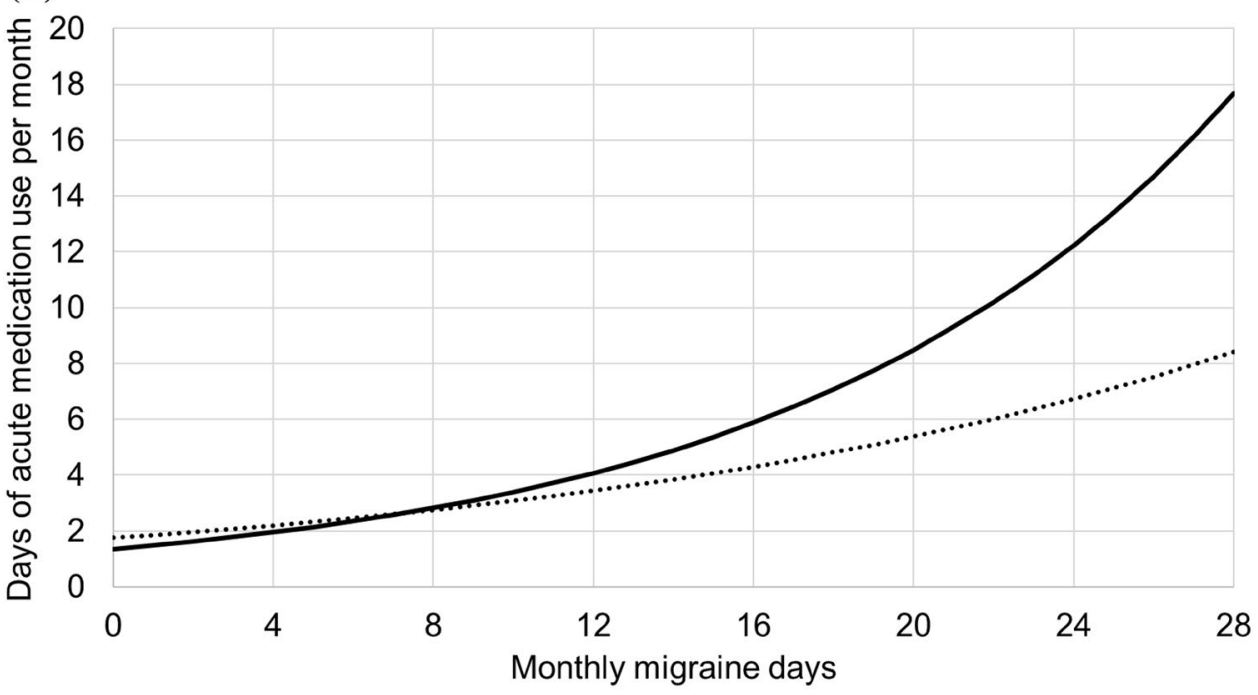

—Migraine specific medication $\quad$...... Non-migraine specific medication

(B)

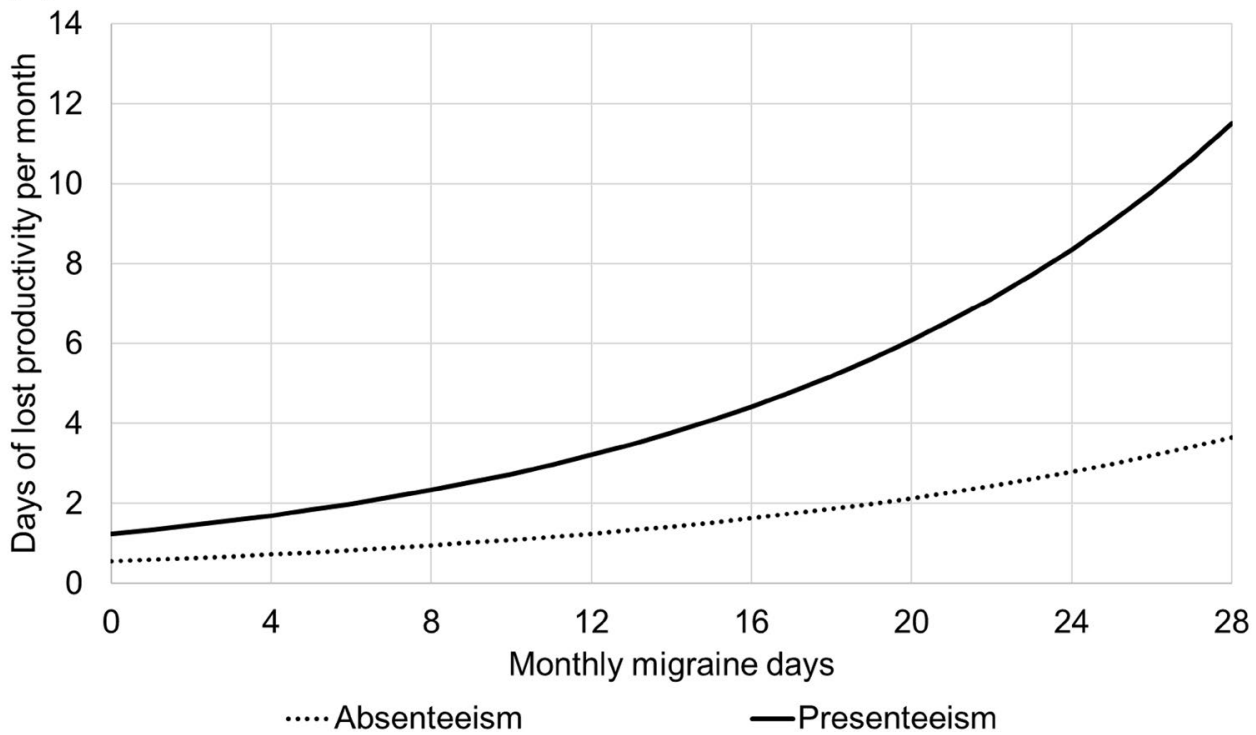


greater use of AM per migraine day for patients with higher MMD.

\subsection{Productivity Losses}

The predicted numbers of days of lost and reduced productivity by MMD are presented in Fig. 1b and Online Resource 3. Details of the regression model are presented in Online Resource 5. A patient experiencing $8 \mathrm{MMD}$ is predicted to have 0.95 (95\% CI 0.94-0.96) absenteeism days and 2.34 (95\% CI 2.32-2.35) presenteeism days per month. Based on the average wage, these productivity losses are expected to result in average costs per month of US\$197.52 (range US\$196.38-US\$198.65) and US\$242.86 (range US\$241.46-US\$244.26), respectively. A patient with CM with $18 \mathrm{MMD}$ is predicted to have 1.86 (95\% CI 1.85-1.87) absenteeism days and 5.18 (95\% CI 5.15-5.21) presenteeism days per month. These productivity losses are expected to result in average costs per month of US $\$ 387.25$ (range US\$385.03-US\$389.48) and US\$538.81 (range US\$535.71-US\$541.91), respectively.

As MMD increases, predicted loss of productivity, due to absenteeism and presenteeism, also increases. The relationship between MMD and productivity loss is such that each incremental MMD results in greater marginal absenteeism and presenteeism days. This is consistent with previous research which showed disproportionately higher indirect costs in patients with CM than in patients with EM [9]. Most days of lost productivity were presenteeism days.

\section{Discussion}

Accurate quantification of AM costs and productivity losses is essential when assessing the economic impact of migraine and cost-effectiveness of migraine prevention. Indirect costs are substantial and have been considered in previous studies of the cost burden of migraine.

The zero-inflated regression models applied here account for the proportions of patients in the clinical studies who reported no AM use or productivity losses. The zero-inflation enables the model to account for the quantity of zero responses, whilst accurately representing the average outcomes of the non-zero responses.

The regression predictions show that as MMD rises, the expected AM use and productivity loss also increases, but the relationship is non-linear. By lowering the MMD of patients, migraine preventives may reduce lost productive time and the requirement for AM. As these relationships are non-linear, considering only mean MMD in economic evaluations may misrepresent outcomes of patient cohorts as the impact of each additional migraine day is not constant.
It is therefore important that the distribution of patients by MMD is accounted for when estimating outcomes of patients with migraine.

The study has several limitations. MIDAS data from the clinical studies only capture workplace productivity losses among employed patients, who can quantify the number of work days impacted by their migraines. Individuals who are unemployed owing to migraine would report no absenteeism or presenteeism using this measure. Therefore, productivity losses of patients with migraine could be underestimated in this study, especially for those with higher MMD. Furthermore, MIDAS data from the CM study required conversion to generate reported productivity losses per month from the 3-monthly observations reported. As patients may have responded differently to a questionnaire with a 1-month recall period compared with a 3-month recall period, this adjustment could have impacted estimated productivity losses.

The accuracy of the estimates of presenteeism presented here is limited by the absence of information on the degree of productivity loss per presenteeism day. The MIDAS questionnaire does not use a scale to measure the proportion of time lost due to migraine. As the minimum impact (50\% lost productivity) was used to estimate the cost of presenteeism, this bias is expected to underestimate the costs. A further limitation of using the MIDAS questionnaire is the lack of information regarding the type of work, which introduces two additional issues. First, occupation and sector will impact the median wage of patients, which may be different to the overall median assumed in this analysis. Second, patients with flexible or team-based work may report different productivity losses, based on their ability to make up for lost work after the migraine attack ends or other team members' ability to compensate for lost time.

There are also limitations to the analytical methods applied. The requirements for zero-inflation and non-linear effects prevented the inclusion of subject-level random effects to account for correlation between the multiple observations reported by each patient. Non-linear, mixed effects regressions able to model zero-inflated data are not routinely accessible or reproducible in standard software packages. Continued research should seek to account for repeated observations by including random effects at the patient level. Finally, outcomes were not stratified by treatment group. In addition to the MMD reduction, active treatment with erenumab may impact the severity and duration of migraines, which may also impact productivity losses and AM use compared with placebo. Future research should aim to compare the outcomes of placebo and actively treated patients, and assess generalizability to inform economic evaluations. 
This analysis shows how lowering MMD via successful prevention can result in lower AM use and productivity losses, and provides a framework for translating clinical endpoints in migraine to potential economic savings.

Data Availability Statement Qualified researchers may request data from Amgen clinical studies. Complete details are available at the following: http://www.amgen.com/datas haring.

Acknowledgements We acknowledge medical writing support by Sinéad Flannery PhD of Oxford PharmaGenesis, Oxford, UK.

Author Contributions All authors were involved in the conception and design of the study. All authors were involved with analysis and interpretation of the data. JKP, GLDT and GV were involved in the patient data collection and acquisition of the data. All authors contributed to the drafting of the manuscript and reviewed and approved the final version.

\section{Compliance with Ethical Standards}

Ethics approval and consent to participate The three trials used in this study were registered with ClinicalTrials.gov, numbers NCT02456740, NCT02483585 and NCT02066415, as previously reported [14, 16, 17]. The studies were approved by the Institutional Review Board/ Independent Ethics Committee (IRB/IEC). The participants provided written informed consent before enrolment.

Funding Funding for this support was provided by Amgen (Europe) $\mathrm{GmbH}$.

Conflict of interest JKP and GLDT are Amgen employees. SS and GV are employed by Amgen and have stock in Amgen. RBL is the Edwin S. Lowe Professor of Neurology at the Albert Einstein College of Medicine in New York. He receives research support from the NIH: 2PO1 AG003949 (Program Director), 5U10 NS077308 (PI), 1RO1 AG042595 (Investigator), RO1 NS082432 (Investigator), K23 NS09610 (Mentor), K23AG049466 (Mentor). He also receives support from the Migraine Research Foundation and the National Headache Foundation. He serves on the editorial board of Neurology and as senior advisor to Headache. He has reviewed for the National Institute on Aging and National Institute of Neurological Disorders and Stroke; holds stock options in eNeura Therapeutics and Biohaven Holdings; and serves as a consultant and/or advisory board member or has received honoraria from the American Academy of Neurology, Alder, Allergan, American Headache Society, Amgen, Autonomic Technologies, Avanir, Biohaven, Biovision, Boston Scientific, Dr. Reddy's, Electrocore, Eli Lilly, eNeura Therapeutics, GlaxoSmithKline, Merck, Pernix, Pfizer, Supernus, Teva, Trigemina, Vector, and Vedanta. He receives royalties from Wolff's Headache, 8th Edition, Oxford Press University, 2009, Wiley and Informa.

Open Access This article is distributed under the terms of the Creative Commons Attribution-NonCommercial 4.0 International License (http://creativecommons.org/licenses/by-nc/4.0/), which permits any noncommercial use, distribution, and reproduction in any medium, provided you give appropriate credit to the original author(s) and the source, provide a link to the Creative Commons license, and indicate if changes were made.

\section{References}

1. Charles A. Migraine. N Engl J Med. 2017;377:1698-9.

2. Headache Classification Committee of the International Headache Society (IHS). The International Classification of Headache Disorders, 3rd edition. Cephalalgia. 2018;38:1-211.

3. Haut SR, Bigal ME, Lipton RB. Chronic disorders with episodic manifestations: focus on epilepsy and migraine. Lancet Neurol. 2006;5:148-57.

4. Katsarava Z, Buse DC, Manack AN, Lipton RB. Defining the differences between episodic migraine and chronic migraine. Curr Pain Headache Rep. 2012;16(1):86-92.

5. Lipton RB, Silberstein SD. Episodic and chronic migraine headache: breaking down barriers to optimal treatment and prevention. Headache. 2015;55(Suppl 2):103-22 (quiz 23-6).

6. Blumenfeld AM, Varon SF, Wilcox TK, Buse DC, Kawata AK, Manack A, et al. Disability, HRQoL and resource use among chronic and episodic migraineurs: results from the International Burden of Migraine Study (IBMS). Cephalalgia. 2011;31:301-15.

7. Bonafede M, Sapra S, Shah N, Tepper S, Cappell K, Desai P. Direct and indirect healthcare resource utilization and costs among migraine patients in the United States. Headache. 2018; https://doi.org/10.1111/head.13275.

8. Stokes M, Becker WJ, Lipton RB, Sullivan SD, Wilcox TK, Wells L, et al. Cost of health care among patients with chronic and episodic migraine in Canada and the USA: results from the International Burden of Migraine Study (IBMS). Headache. 2011;51:1058-77.

9. Munakata J, Hazard E, Serrano D, Klingman D, Rupnow MF, Tierce $\mathrm{J}$, et al. Economic burden of transformed migraine: results from the American Migraine Prevalence and Prevention (AMPP) study. Headache. 2009;49:498-508.

10. Lafata JE, Moon C, Leotta C, Kolodner K, Poisson L, et al. The medical care utilization and costs associated with migraine headache. J Gen Intern Med. 2004;19:1005-7.

11. Antonaci F, Dumitrache C, De Cillis I, Allena M. A review of current European treatment guidelines for migraine. J Headache Pain. 2010;11:13-9.

12. Goldberg LD. The cost of migraine and its treatment. Am J Manag Care. 2005;11:S62-7.

13. Bigal ME, Walter S, Rapoport AM. Calcitonin gene-related peptide (CGRP) and migraine current understanding and state of development. Headache. 2013;53:1230-44.

14. Goadsby PJ, Reuter U, Hallstrom Y, Broessner G, Bonner JH, Zhang F, et al. A controlled trial of erenumab for episodic migraine. N Engl J Med. 2017;377:2123-32.

15. Food and Drug Administration. 2018. Aimovig (erenumab). Prescribing Information. https://www.accessdata.fda.gov/drugsatfda _docs/label/2018/761077s000lbl.pdf. Accessed 21 June 2018.

16. Dodick DW, Ashina M, Brandes JL, Kudrow D, Lanteri-Minet $\mathrm{M}$, Osipova V, et al. ARISE: a phase 3 randomized trial of erenumab for episodic migraine. Cephalalgia. 2018. https://doi. org/10.1177/333102418759786.

17. Tepper S, Ashina M, Reuter U, Brandes JL, Dolezil D, Silberstein $S$, et al. Safety and efficacy of erenumab for preventive treatment of chronic migraine: a randomised, double-blind, placebo-controlled phase 2 trial. Lancet Neurol. 2017;16:425-34.

18. Bonafede M, Sapra S, Tepper SJ, Cappell KA, Desai P. Healthcare costs and utilization and medication treatment patterns among migraine patients: a retrospective analysis. Headache. 2017;57:211-211.

19. Graubard BI, Korn EL. Predictive margins with survey data. Biometrics. 1999;55:652-9.

20. Bigal ME, Rapoport AM, Lipton RB, Tepper SJ, Sheftell FD. Assessment of migraine disability using the migraine disability 
assessment (MIDAS) questionnaire: a comparison of chronic migraine with episodic migraine. Headache. 2003;43:336-42.

21. Stewart WF, Lipton RB, Kolodner K. Migraine disability assessment (MIDAS) score: relation to headache frequency, pain intensity, and headache symptoms. Headache. 2003;43:258-65.
22. Bureau of Labor Statistics. Private Sector Dec 2016. https://www. bls.gov/news.release/archives/realer_01182017.pdf. Accessed 23 Mar 2017. 